\title{
Mangifera indica Mediated Synthesis of Silver Nanoparticles as An Efficient Electrochemical Sensor for The Detection of Ascorbic Acid
}

\author{
Solomon A. Mamuru ${ }^{1, *}$, Olushola O. Joseph ${ }^{1}$, Moorey B. Dalen², Iliya Kaigamma ${ }^{1}$, Anthonia E. Eseyin ${ }^{2}$ \\ ${ }^{1}$ Electrochemical NanoLab, Department of Chemistry, Adamawa State University, Mubi, Nigeria. \\ ${ }^{2}$ Department of Chemistry, University of Jos, Jos, Nigeria.
}

\section{A R T I C L E D E T A I L S}

Article history:

Received 20 November 2018

Accepted 21 May 2019

Available online 01 August 2019

\section{Keywords:}

Silver Nanoparticles

Mangifera indica

Ascorbic Acid Oxidation

\begin{abstract}
A B S T R A C T
The biosynthesis of silver nanoparticles using Mangifera indica leaf extract as reducing agent was successfully carried out. The usual microscopic and spectroscopic techniques such as UV-vis., FTIR, XRD, EDX, and SEM were used to confirm the formation of nanoparticles. The silver nanoparticles absorb light at wavelength of approximately $425 \mathrm{~nm}$ as confirmed by UV- visible spectroscopy. Optical property showed a colour change from colourless solution of silver nitrate to yellowish brown after addition of the plant extract. The FTIR confirmed the possible biomolecule responsible for the reduction of the silver nitrate solution as belonging to the amide and ester group. Interrogation of Pt/AgNP electrode for ascorbic acid oxidation using cyclic voltammetry suggests that the silver nanoparticles can be a promising candidate as biosensor for ascorbic acid detection.
\end{abstract}

\section{Introduction}

The application of nanoscale materials and structures, usually ranging from 1 to $100 \mathrm{~nm}$, is an emerging area of nanoscience and nanotechnology [1]. Plant mediated synthesis of metallic nanoparticles is in increasing commercial demand due to the wide applicability in various areas such as electronics, catalysis, energy, cosmetics and medicine. Production of nanoparticles can be achieved through different methods. Chemical approaches are the most popular methods for the production of nanoparticles. However, some chemical methods cannot avoid the use of toxic chemicals in the synthesis protocol. Since noble metal nanoparticles such as that of gold, silver and platinum are widely applied to human contacting areas. There is a growing need to develop environmentally friendly processes of nanoparticles synthesis that do not use toxic chemicals [2]. Biological methods of nanoparticles synthesis using microorganisms [3], enzyme [4] and plant or plant extracts [5] have been suggested as possible eco-friendly alternatives to chemical and physical methods. Although many plants extract, some of which include, Azadirachta indica, Aloe barbadensis, Musa paradisiaca, Saccharum officinarum, Basella alba, Mangifera indica, Oryza sativa and others [6-12] have been successfully used in the synthesis of metal nanoparticles. This study seeks to synthesize silver nanoparticles using leaves extract of Mangifera indica and show its electrochemical behavior towards the detection of ascorbic acid.

\section{Experimental Methods}

\subsection{Materials}

Mangifera indica leaves; Autolab PGSTAT 302N; Hanna Instrument 2211 pH meter; silver nitrate; sodium hydroxide; sodium chloride, disodium hydrogen phosphate; sodium dihydrogen phosphate; ethanol; ascorbic acid (tablet); Jenway 6400 UV-visible spectrophotometer; Perkin Elmer Frontier Fourier Transform Infrared Spectroscopy and Ultrasonic bath were employed for the study.

\subsection{Preparation of Leaf Extract}

$10 \mathrm{~g}$ fresh leaves of Mangifera indica plant was washed with distilled water to remove dust and cut into pieces. It was placed in $250 \mathrm{~mL}$
Erlenmeyer flask and boiled with distilled water for 20 minutes then the mixture was allowed to cool at room temperature. The solution was then filtered with Whatman filter paper No.1 to obtain the Mangifera indica leaf extract which was later used as a reducing agent for the bio-reduction of silver nitrate.

\subsection{Synthesis of Silver Nanoparticles}

The synthesis procedure of S.A. Mamuru et al. [5] was adopted. $0.042 \mathrm{~g}$ of silver nitrate was weighed with a top load balance and dissolved in 100 $\mathrm{mL}$ distilled water to prepare a $0.001 \mathrm{M}$ silver nitrate solution. $60 \mathrm{~mL}$ aqueous solution of $1 \mathrm{mM}$ of silver nitrate was reduced using $5 \mathrm{~mL}$ of the leave extract at room temperature for 10 minutes; change in colour of the solution indicated the formation of silver nanoparticles. The product was centrifuged at $3000 \mathrm{rpm}$ for 10 minutes, then washed with ethanol and distilled water and allowed to dry at room temperature.

\subsection{Ultraviolet-Visible Spectroscopy and FTIR Investigation}

The presence of the silver nanoparticles synthesized was confirmed by sampling the aqueous component using UV-visible spectrophotometer Distilled water was used as a blank. Absorbance measurement was carried out at a wavelength of 300-800 nm using UV-visible spectrophotometer at a scan interval of $5 \mathrm{~nm}$ in order to obtain the UV-visible spectrum of the nanoparticles. The biomolecule responsible for the reduction of the AgNPs was determined using Perkin Elmer Fourier Transform Infrared (FT-IR) spectroscopy.

\subsection{Preparation of Phosphate Buffer Saline Solution}

$250 \mathrm{~mL}$ phosphate buffer saline solution was prepared by weighing $2.725 \mathrm{~g}$ of anhydrous sodium phosphate dibasic, $0.8 \mathrm{~g}$ of sodium phosphate monobasic and $22.5 \mathrm{~g}$ of sodium chloride. They were poured into a $250 \mathrm{~mL}$ volumetric flask and distilled water was added to the mark.

\subsection{Electrochemical Study}

All electrochemical measurements were performed with an Autolab PGSTAT 302N Potentiostat driven by NOVA software version 1.9. A threeelectrode system was employed for the study. The counter electrode was a platinum wire, $\mathrm{Ag} / \mathrm{AgCl} 3 \mathrm{M} \mathrm{KCl}$ was used as reference electrode and working electrode was Ag nanoparticle modified platinum electrode. 


\subsubsection{Electrode Preparation}

$10 \mu \mathrm{L}$ of the Ag nanoparticle solution was placed on the surface of an already polished and ultrasonically rinsed platinum electrode $(3 \mathrm{~mm}$ diameter) using a micropipette and allowed to dry at room temperature to prepare the Platinum-Silver nanoparticle electrode (Pt/AgNP). Electrochemical properties of the bare electrode and modified electrode were studied using phosphate buffer saline solution as the blank.

\subsubsection{Preparation of 0.001 M Ascorbic Acid in Phosphate Buffer Solution}

$0.315 \mathrm{~g}$ of ascorbic acid tablet was dissolved in $50 \mathrm{~mL}$ of the already prepared phosphate buffer saline solution to prepare $0.001 \mathrm{M}$ ascorbic acid. $0.335 \mathrm{~g}$ of the ascorbic acid tablet contains $0.01 \mathrm{~g}$ ascorbic acid hence $0.315 \mathrm{~g}(0.009 \mathrm{~g}$ ascorbic acid) of the tablet is needed to prepare $50 \mathrm{~mL}$ $0.001 \mathrm{M}$ ascorbic acid.

\subsubsection{Ascorbic Acid Detection}

Ascorbic acid oxidation was carried out using cyclic voltammetry (CV). $0.001 \mathrm{M}$ ascorbic acid in phosphate buffer saline solution and phosphate buffer saline solution were used as the main and blank solutions, respectively.

\section{Results and Discussion}

\subsection{Reaction Colour and UV-Visible Spectroscopy}

Leaf extract of Mangifera indica was used for the reduction of silver nitrate solution to form AgNPs. The reduction of silver ion into silver nanoparticles during exposure to the leaf extract of Mangifera indica was monitored by change in colour. A mixture of colourless solution of silver nitrate and the light yellow colour of Mangifera indica solution changed into brown which indicates the formation of silver nanoparticles, as shown in Fig. 1. The formation of brown colour was developed between 5-30 minutes. The intensity of the colour increases with an increase in the contact time. This colour was basically due to surface plasmon resonance of the silver nanoparticles which is attributed to the collective oscillation of conduction band electron in response to the electrical field of the electromagnetic radiation of light. This phenomenon is absent at the scales of atoms or the bulk material $[13,14]$.

UV-visible spectroscopy was used for further investigation of AgNPs synthesis in this work. As can be seen in Fig. 1, the maximum absorbance peak appears at approximately $425 \mathrm{~nm}$, which is within the range for absorbance of metallic silver. Furthermore, the band gap energy was estimated using the Planck's equation [15], Band gap energy $(\mathrm{E})=\mathrm{hC} / \lambda$, where, $\mathrm{h}=$ Planck's constant $=6.626 \times 10^{-34} \mathrm{Js}, \mathrm{C}=$ speed of light $=3.0 \times 10^{8}$ $\mathrm{m} / \mathrm{s}, \lambda=$ cut off wavelength $=425 \times 10^{-9} \mathrm{~m}$. The band gap energy for metallic silver was calculated to be ca. $2.93 \mathrm{eV}$ using a conversion factor of $1 \mathrm{eV}$ equivalent to $1.6 \times 10^{-19}$ Joules.

The "band gap" refers to the energy difference between the top of the valence band to the bottom of the conduction band; electrons are able to jump from one band to another. In order for an electron to jump from a valence band to a conduction band, it requires a specific minimum amount of energy for the transition, the band gap energy. The band gap is a major factor determining the electrical conductivity of a solid. Substances with large band gaps $(>4 \mathrm{eV})$ are generally insulators, those with smaller band gaps $(<3 \mathrm{eV})$ are semiconductors, while conductors either have very small band gaps or none, because the valence and conduction bands overlap [16, 17].

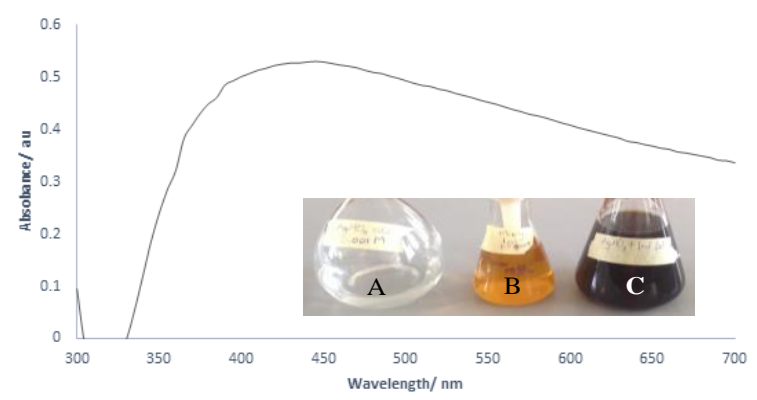

Fig. 1 UV- visible absorption spectrum of Ag nanoparticles synthesized using Mangifera indica leaf extract. (Inset) reaction colour; silver nitrate (A), Mangifera indica leaves extract (B) and silver nanoparticles (C)

\subsection{FTIR Spectroscopy Analysis}

The Fourier transform infra-red spectroscopy analysis was performed to identify the possible biomolecule responsible for the bioreduction of https://doi.org/10.30799/jacs.200.19050204
$\mathrm{Ag}^{+}$ions and capping of the reduced silver nanoparticles synthesized using Mangifera indica leaves. Fig. 2 shows the comparative spectra of Mangifera indica and silver nanoparticles.

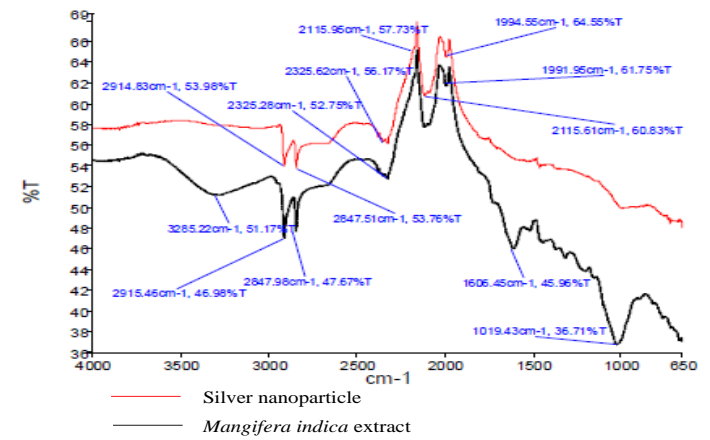

Fig. 2 Comparative FTIR spectroscopy spectrum of Mangifera indica leaf extracts and silver nanoparticles

The result obtained for Mangifera indica leaves gave IR bands, at 3285 , $2915,2847,2325,2115,1991,1606$ and $1019 \mathrm{~cm}^{-1}$. The IR bands for silver nanoparticles were observed at the following bands 2915, 2847, 2325, 2115 and $1991 \mathrm{~cm}^{-1}$. The bands for Mangifera indica leaves and silver nanoparticles are associated with the following functional groups as presented in Tables 1 and 2. From the bands obtained for Mangifera indica leaves and silver nanoparticles, the bands at $3285 \mathrm{~cm}^{-1}, 1606 \mathrm{~cm}^{-1}$ and $1019 \mathrm{~cm}^{-1}$ are the ones in Mangifera indica leaves that are absent in silver nanoparticles. These functional groups are identified as primary amine, aliphatic, R-CHR-NH2 [18]. Therefore, it can be suggested that the biomolecule responsible for the reduction of $\mathrm{Ag}^{+}$to silver nanoparticles is the group of aliphatic primary amines.

Table 1 FTIR spectroscopy analysis of Mangifera indica leaves extract

\begin{tabular}{llll}
\hline S.No. & Vibrational group & Band frequency, $\mathrm{cm}^{-1}$ & Functional group remark \\
\hline 1 & Alcohol $-\mathrm{OH}$ & $3285.22(\mathrm{~s})$ & stretch, H-bonded \\
2 & Alkane C-H & $2915.46(\mathrm{~s})$ & stretch \\
3 & Aldehyde $=\mathrm{C}-\mathrm{H}$ & $2847.98(\mathrm{~m})$ & stretch \\
4 & C $\equiv$ N & $2325.28(\mathrm{~s})$ & stretch \\
5 & Alkyne $-\mathrm{C} \equiv \mathrm{C}-$ & $2115.95($ variable) & stretch \\
6 & Amide N-H & 1606.45 & bending \\
7 & Ester C-O & 1019.43 & stretch \\
\hline
\end{tabular}

Table 2 FTIR spectroscopy analysis of AgNP synthesized from Mangifera indica leaves extract

\begin{tabular}{llll}
\hline S.No. & Vibrational group & Band frequency, $\mathrm{cm}^{-1}$ & Functional group remark \\
\hline 1 & Alkane C-H & $2915.46(\mathrm{~s})$ & stretch \\
2 & Aldehyde $=\mathrm{C}-\mathrm{H}$ & $2847.98(\mathrm{~m})$ & stretch \\
3 & C $\equiv$ N & $2325.28(\mathrm{~s})$ & stretch \\
4 & Alkyne $-\mathrm{C} \equiv \mathrm{C}-$ & $2115.95($ variable) & stretch \\
\hline
\end{tabular}

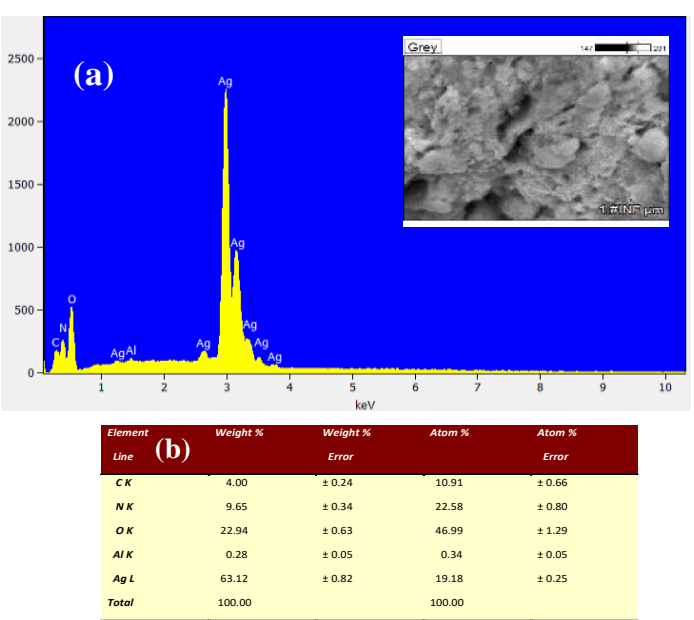

Fig. 3 Energy dispersive spectroscopy elemental analysis of Mangifera indica synthesized silver nanoparticles

\subsection{Energy Dispersive Spectroscopy (EDS)}

Fig. 3(a) presents an X-ray elemental microanalysis captured from a region observed by SEM (inset) from a drop of the resulting silver nanoparticle dried over a carbon surface. The most prominent peak obtained at the energy of $3 \mathrm{eV}$ corresponds to that of silver indicating the 
reduction of silver ions to element of silver. This value corroborates that obtained using Planck's equation. Other weak peaks for elements C, O, N and $\mathrm{Al}$ were also obtained. Fig. 3(b) quantitatively analysed the elements and measure them as $63 \% \mathrm{Ag}, 23 \% \mathrm{O}, 10 \% \mathrm{~N}, 4 \% \mathrm{C}$ and $0.28 \% \mathrm{Al}$.

\subsection{X-Ray Diffraction (XRD)}

The crystalline nature of the synthesized silver nanoparticles was further confirmed from their powder X-ray diffraction (XRD) patterns, as shown in Fig. 4. The presence of four lattice planes viz, (111), (200), (220), and (311) confirmed the formation of face-centered cubic (FCC) crystal structure of silver nanoparticles, and these lattice planes were associated with the diffraction peaks at ca $38.08^{\circ}, 44.04^{\circ}, 64.49^{\circ}$ and $77.34^{\circ}$. These results confirmed the formation of metallic silver (JCPDS 04-0783) [19].

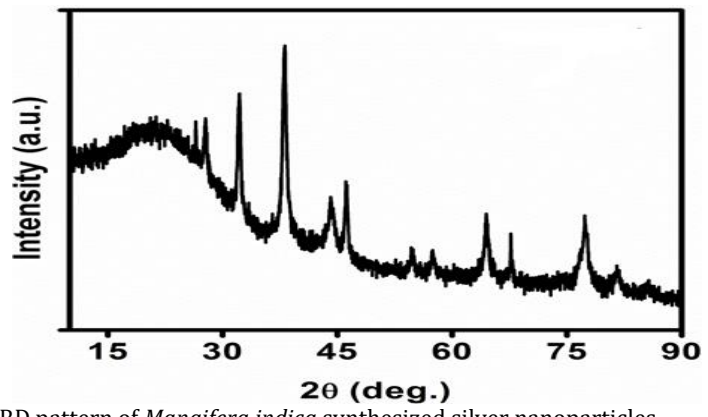

Fig. 4 XRD pattern of Mangifera indica synthesized silver nanoparticles

\subsection{Scanning Electron Microscopy}

Fig. 5 shows the SEM micrograph of Mangifera indica synthesized silver nanoparticles as spherical but twisted agglomerated particles.

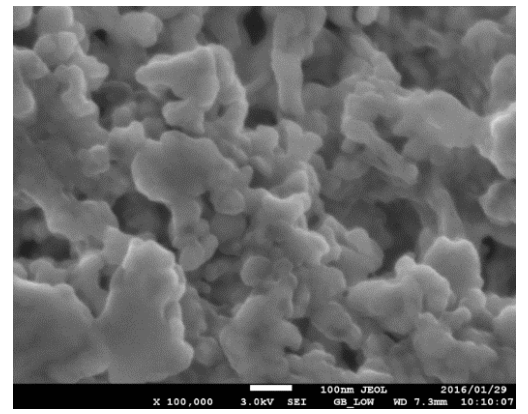

Fig. 5 SEM micrograph of Mangifera indica synthesized silver nanoparticles
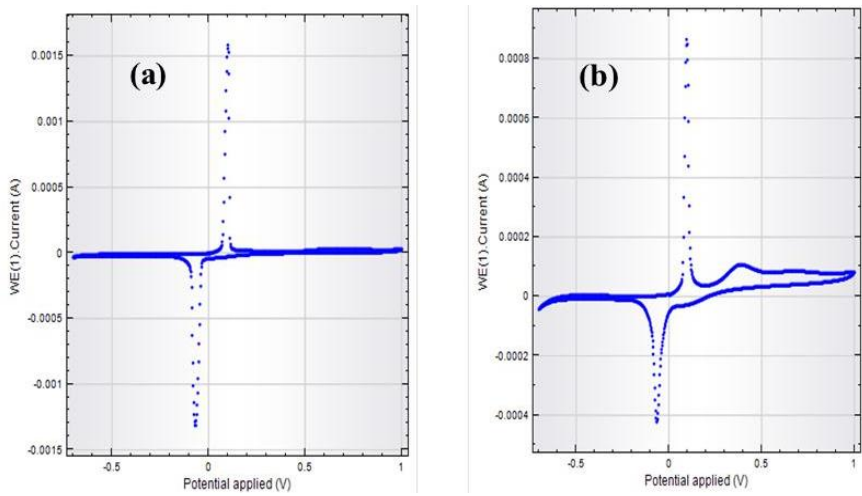

Fig. 6 Comparative cyclic voltammetric evolutions of Pt/AgNP electrodes in (a) phosphate buffer saline solution and (b) phosphate buffer saline $+0.001 \mathrm{M}$ ascorbic acid

\subsection{Electrocatalytic Oxidation of Ascorbic Acid}

Fig. 6 shows the electro-catalytic behavior of Pt/AgNP for ascorbic acid electro-oxidation in $0.001 \mathrm{M}$ ascorbic acid + phosphate buffer saline solution. Pair of oxidation peaks was observed at $0.1 \mathrm{~V}$ and $-0.08 \mathrm{~V}$ in Fig. 6(a) which may be attributed to the electroactive nature of the modified electrode. The same peaks were observed in Fig. 6(b) however, a new oxidation peak was observed at ca. $0.385 \mathrm{~V}$ which indicates the oxidation of ascorbic acid as such peak was not observed in the absence of ascorbic acid as shown in Fig. 6(a). Khalilzadeh et al. [20] and Noroozifar et al. [21] reported oxidation of ascorbic acid at $0.483 \mathrm{~V}$ and $0.442 \mathrm{~V}$ vs. $\mathrm{Ag} \mid \mathrm{AgCl}$, respectively. In this work, ascorbic acid was observed at approximately
$100 \mathrm{mV}$ less than the other workers implying that the Pt/AgNP electrode is a better detector of ascorbic acid.

\section{Conclusion}

Silver nanoparticles were synthesized, using the leaf extract of Mangifera indica. The synthesized AgNP formation was confirmed by the change in colour of the silver nitrate nanoparticles from colourless to yellowish brown. The nanoparticle was characterized by Ultraviolet visible spectroscopy, SEM, EDX and XRD. FT-IR spectroscopy was used to identify the biomolecules responsible for the bio-reduction. The synthesized silver nanoparticles showed an oxidation peak potential of $0.385 \mathrm{~V}$ when used as modified electrode in the detection of ascorbic acid. No peak was observed when used in phosphate buffer solution. Thus, AgNPs exhibited a catalytic property towards the oxidation of ascorbic acid and can possibly be used as a sensor to indicate the presence of ascorbic acid.

\section{Acknowledgement}

The authors will like to thank Adamawa State University for providing the facilities and resources for carrying out this work.

\section{References}

[1] K. Gopinath, S. Gowri, A. Arumugam, Phytosynthesis of silver nanoparticles using Pterocarpus santalinus leaf extract and their antibacterial properties, J. Nanostruct. Chem. 3(68) (2013) 1-7.

[2] K. Satyavani, T. Ramanathan, S. Gurudeeban, Green synthesis of silver nanoparticles by using stem derived callus extract of bitter apple (Citrullus colocynthis), Dig. J. Nanomater. Biostruct. 6(3) (2011) 1019-1024.

[3] K.C. Bhainsa, S.F. D'Souza, Extracellular biosynthesis of silver nanoparticles using the fungus Aspergillus fumigatus, Colloids Surf. B 47 (2006) 160-164.

[4] J.M. Palomo, M. Filice, Biosynthesis of metal nanoparticles: Novel efficient heterogeneous nanocatalysts, Nanomater. 6(84) (2016) 1-16.

[5] S.A. Mamuru, N. Jaji, Voltammetric and impedimetric behavior of phytosynthesized nickel nanoparticles, J. Nanostruct. Chem. 5 (2015) 347-356.

[6] M. Mankad, G. Patil, D. Patel, P. Patel, A. Patel, Comparative studies of sunlight mediated green synthesis of silver nanoparticles from Azadirachta indica leaf extract and its antibacterial effect on Xanthomonas oryzae pv. oryzae, Arab. J. Chem. (2018) In-Press. Doi: https://doi.org/10.1016/j.arabjc.2018.07.016.

[7] M.M.H. Ibrahim, Green synthesis and characterization of silver nanoparticles using banana peel extract and their antimicrobial activity against representative microorganisms J. Radiat. Res. Appl. Sci. 8(3) (2015) 265-275.

[8] E. Vélez, G. Campillo, G. Morales, C. Hincapié, J. Osorio, O. Arnache, Silver nanoparticles obtained by aqueous or ethanolic Aloe vera extracts: An assessment of the antibacterial activity and mercury removal capability, J. Nanomater. 2018 (2018) 7215210:1-7.

[9] P. Chaudhari, S. Masurkar, S.P. Kamble, Biosynthesis of silver nanoparticles using Saccharum officinarum and its antimicrobial activity, Micro Nano Lett. 7(7) (2012) 646-650.

10] K.L. Niraimathi, R. Lavanya, V. Sudha, P. Brindha, Green synthesis and characterization of silver nanoparticles from aqueous extract of Basella Alba and their In-Vitro antioxidant potentials, Int. J. Pharm. Sci. 6(10) (2014) 1-16.

[11] M. Reenal, K. Iruthaya, S.S. Kalai, Green synthesis and antibacterial activity of silver nanoparticles using Oryza sativa husk extract, Inter. Res J. Environ. Sci. 4(5) (2015) 68-72.

[12] D. Sundeep, T.V. Kumar, P.S. Subba Rao, R.V.S.S.N. Ravikumar, A.G. Krishna Green synthesis and characterization of $\mathrm{Ag}$ nanoparticles from Mangifera indica leaves for dental restoration and antibacterial applications, Prog. Biomater. 6 (2017) 57-66.

[13] U. Kreibig, M. Vollmer, Optical properties of metal clusters, Springer, Berlin, 1995.

[14] C.F. Bohren, D.R. Huffman, Absorption and scattering of light by small particles, John Wiley, New York, 1983.

[15] R. Ma, V. Sharma, A.F. Baldwin, M. Tefferi, I. Offenbach, et al., Rational design and synthesis of polythioureas as capacitor dielectrics, J. Mater. Chem. A 3 (2015) 14845-14852.

[16] M. Hoffman, S. Martin, W. Choi, D. Bahnemann, Environmental applications of semiconductor photo catalysis, Chem. Rev. 95 (1995) 69-96.

[17] Wikipedia: Bandgap definition and http://en.wikipedia.org/wiki/Bandgap (Accessed on: 19.11.2018)

[18] P. Patnaik, Dean's analytical chemistry handbook, McGraw Hill, NY, USA, 2004

[19] M.N. Alam, S. Das, S. Batuta, D. Mandal, N.A. Begum, Green-nanochemistry for safe environment: bio-friendly synthesis of fluorescent monometallic (Ag and $\mathrm{Au})$ and bimetallic ( $\mathrm{Ag} / \mathrm{Au}$ alloy) nanoparticles having pesticide sensing activity, J. Nanostruct. Chem. 6(4) (2016) 373-395.

[20] M.A. Khalilzadeh, M. Borzoo, Green synthesis of silver nanoparticles using onion extract and their application for the preparation of a modified electrode for determination of ascorbic acid, J. Food Drug Anal. 24 (2016) 796 -803.

[21] M. Noroozifar, M. Khorasani-Motlagh, H. Tavakkoli, Electrochemical determination of ascorbic acid using modified glassy carbon electrode by multiwall carbon nanotube-nafion in chloroacetic acid media, Asian J. Chem. 25(1) (2013) 119-124. 